COMMUNICATIONS IN

ANALYSIS AND GEOMETRY

Volume 13, Number 1, 89-111, 2005

\title{
Nonexistence of Invariant Rigid Structures and Invariant Almost Rigid Structures
}

\author{
E. Jerome Benveniste And David Fisher
}

We prove that certain volume preserving actions of Lie groups and their lattices do not preserve rigid geometric structures in the sense of Gromov. The actions considered are the "exotic" examples obtained by Katok and Lewis and the first author, by blowing up closed orbits in the well known actions on homogeneous spaces. The actions on homogeneous spaces all preserve affine connections, whereas the action along the exceptional divisor preserves a projective structure. The fact that these structures cannot in some way be "glued together" to give a rigid structure on the entire space is not obvious.

We also define the notion of an almost rigid structure. The paradigmatic example of a rigid structure is a global framing and the paradigmatic example of an almost rigid structure is a framing that is degenerate along some exceptional divisor. We show that the actions discussed above do possess an invariant almost rigid structure.

Gromov has shown that a manifold with rigid geometric structure invariant under a topologically transitive group action is homogeneous on an open dense set. How generally this open dense set can be taken to be the entire manifold is an important question with many dynamical applications. Our results indicate one way in which the geometric structure cannot degenerate off the open dense set.

\section{Introduction.}

Let $G$ be a semisimple Lie group with all simple factors of real rank at least 2 and $\Gamma$ a lattice in $G$. Zimmer and Gromov have conjectured that actions of $G$ or $\Gamma$ on compact manifolds that preserve unimodular rigid geometric structures, for example an affine connection and a volume form, should be "essentially classifiable" $[12,18,19]$. However, there are "exotic" actions of these groups on compact manifolds where it had been unknown if an invariant rigid geometric structure existed. These "exotic" actions are on manifolds with non-linear fundamental group and the actions admit 
non-trivial deformations, both of which cast doubt on the possibility of the desired classification. The purpose of this article is to show that these actions do not admit invariant rigid geometric structures. The simplest action we consider is obtained by modifying the action of $S L_{n}(\mathbb{Z})$ on $\mathbb{T}^{n}$. In [14], Katok and Lewis construct a new action from this action by "blowing up" the origin in the algebro-geometric sense. Throughout this article, in keeping with standard terminology from algebraic geometry, we will call the submanifold obtained from the point by blowing up the exceptional divisor. Katok and Lewis then further modify this action so that it is volume preserving. It is claimed in that paper that there is no invariant rigid geometric structure, though no argument is given. What is fairly clear is that the affine connection on $\mathbb{T}^{n}$ cannot be extended over the exceptional divisor.

In [2], the first author generalizes the Katok-Lewis construction to show that one can construct other examples by blowing up closed orbits. Let $G$ be a simple group, $H$ a semisimple group and $\Lambda$ a lattice in $H$. Then $\mathrm{G}$ acts on $H / \Lambda$. Given appropriate algebraic conditions, there will be many closed $G$ orbits $X$ for this action, and one can "blow-up" the normal directions to $X$ along $X$. (More generally, one could blow up along closed $G$ invariant submanifolds.) For the specific examples considered in [2], it is shown that there is no invariant affine connection.

In this paper, we prove there is no rigid invariant $A$-structure in the sense of Gromov on any of these examples.

Sketch of proof: The idea of the proof is very simple, and we outline it here in the case of the action of $S L(n, \mathbb{Z})$ on the torus with the origin blown-up. This action agrees with the standard action on the torus on an open dense invariant set. Assume there is an invariant rigid structure $\omega$. We obtain a contradiction in two steps. First we show that, away from the exceptional divisor, any local isometry of the standard affine structure on $\mathbb{T}^{n}$ is a local isometry of $\omega$. This uses Iozzi's Geometric Borel Density Theorem, which is, morally speaking, a form of very strong ergodicity for all measure preserving actions of semisimple Lie groups and their lattices. Note that while this implies that $\omega$ has "at least as many" local symmetries as the standard connection on $\mathbb{T}^{n}$, it does not imply that $\omega$ agrees with the standard connection, even off the exceptional divisor. In the second step, we show that some particular local isometries of the standard affine structure necessarily degenerate badly on the exceptional divisor in a manner that contradicts the rigidity of $\omega$. We now describe each step in slightly greater detail.

In the first use Iozzi's thesis to show the invariance of $\omega$ under $S L_{n}(\mathbb{Z})$ to show that, off the exceptional divisor, the structure is invariant under 
the local action of any (partially defined) affine vector field on the torus. To apply her result, we first lift the action to $\mathbb{R}^{n}$ (technically, $\mathbb{R}^{n}$ with the integers blown-up, but since we work on the open dense set that is the complement of the integers, this is irrelevant). Here the $S L_{n}(\mathbb{Z})$ action extends to a $S L_{n}(\mathbb{R})$ action, and the content of Iozzi's theorem says exactly that $\omega$ is invariant under $S L_{n}(\mathbb{R})$. (See Theorem 4.1 for a precise statement.) Since it is $\mathbb{Z}^{n}$ invariant by construction, it will then be invariant under all of $S L_{n}(\mathbb{R}) \ltimes \mathbb{R}^{n}$. This action does not descend to the torus, but it does descend locally, so we have a large set of vector fields that preserve the structure locally.

The rest of the proof is conceptually simple, but somewhat computationally involved in practice. We use one of the vector fields produced above to show that the structure cannot be rigid at a point on the exceptional divisor. We look at the infinitesimal isometry $\tau$ of the structure defined by a vector field $V$ and look at what happens to $\tau$ as we tend towards the exceptional divisor. By making an appropriate choice of $V$ we can produce an infinitesimal isometry of order $k$ at a point on the exceptional divisor that is trivial up to order $k-1$. This directly contradicts the definition of a rigid geometric structure. The vector field $V$ we construct would vanish to order $k$ at the origin on the torus, were the exceptional divisor not present. We show by explicit computation that the procedure of "blowing-up" reduces this order of vanishing by one.

Remark: Though the main interest of our results is in the case of higher rank groups, there is no rank assumption anywhere in this paper. Our arguments apply equally well to actions of rank 1 simple groups and their lattices and particularly to $S L(2, \mathbb{Z})$ acting on $\mathbb{T}^{2}$ with the origin blown up.

In the other cases we discuss below, we follow essentially the same outline. One can construct somewhat more general examples, which we do not discuss here, since the main obstacle to a more general non-existence result is a generalization of Iozzi's theorem.

The paradigmatic example of a rigid geometric structure on $M$ is a global framing on some higher order frame bundle $P^{k}(M)$. That such a framing is a rigid structure follows easily from the computation that a non-vanishing vector field on $\mathbb{R}$ is a rigid structure. And in fact, any rigid structure defines such a framing. In the last section of this article we define an almost rigid $A$ structure and show that the actions discussed above do admit almost rigid $A$-structures. The paradigmatic example of such a structure is a global framing on $P^{k}(M)$ that degenerates to a subframing over some submanifold of $M$. The simplest such example is a vector field on $\mathbb{R}$ that vanishes at only finitely many points and only to finite order. 
Given a compact manifold $M$ and a rigid geometric structure $\omega$ on $M$. Further assume that the automorphism group of $(M, \omega)$ has a dense orbit. One of the principal results of [12], is that there is an open dense set $U$ in $M$ such that the local isometries of $\omega$ act transitively on $U$. Since the set of local isometries are everywhere finite dimensional, this exhibits a locally homogeneous structure on $U$ of the form $H / L$ where $H$ is a Lie group. The proof of our results involves explicitly identifying a large subgroup of $H$. The proof of the results amount to showing that the homogeneous structure on $U$ cannot deteriorate too badly on $M \backslash U$. Determing when $U=M$ is a question with many potential dynamical applications. It is, for instance, a key step in the celebrated work of Benoist, Foulon, and Labourie [3]. More interesting connections between dynamics and rigid structures have recently been pointed out by Renato Feres [9]. There is also a large literature of alternative expositions of Gromov's proof, see for example $[4,5,6,7,8,16$, 17].

In section 3 we provide two examples of rigid geometric structures on non-homogeneous manifolds. For the first the manifold $N_{1}$ is $\mathbb{R}^{n}$ with the origin blown up, and for the second the manifold $N_{2}$ is the compactification of $N_{1}$ obtained by viewing the complement of the blow up as a subset of the real projective space $P^{n}$. Both rigid structures are invariant under natural actions of $S L_{n}(\mathbb{R})$. This paper grew out of an attempt to use these structures to construct an $S L_{n}(\mathbb{Z})$ invariant rigid structure on the examples of Katok and Lewis. Our proof can be interpreted as showing this to be impossible because the holonomy of the rigid structure on the torus generates too many "extra" local isometries. To illustrate this point, we also exhibit a geometric structure on $N_{2}$ invariant under an action of $S L_{n}(\mathbb{R}) \ltimes \mathbb{R}^{n}$ which preserves the exceptional divisor. While the tautological structure on $N_{2}$ for the $S L_{n}(\mathbb{R})$ action is rigid, the tautological structure for the $S L_{n}(\mathbb{R}) \ltimes \mathbb{R}^{n}$ action is not. This difference is closely related to the fact that the second group has nontrivial unipotent radical, see discussion at the end of section 3.

We would like to thank Renato Feres for many helpful conversations in the early phases of this project. Both authors would like to thank the Newton Institute for it's hospitality at the beginning of this project. The second author was partially supported by NSF grant numbers DMS-9902411 and DMS-0226121 and a PSC-CUNY grant.

\section{Actions.}

In [14], Katok and Lewis describe certain actions of arithmetic lattices in $S L(n, \mathbb{R})$. To fix notation, we will briefly describe their construction. 
Let $\mathbb{T}^{n}$ denote the $n$-dimensional torus, and let $p_{+}$and $p_{-}$denote the points $\left(\frac{1}{2}, 0, \ldots, 0\right)$ and $\left(0, \frac{1}{2}, 0, \ldots, 0\right)$ on $\mathbb{T}^{n}$ (in terms of linear coordinates); then $p_{+}$and $p_{-}$are fixed points for the action of the congruence subgroup $\Gamma_{2} \subset S L(n, \mathbb{Z})$. Let $A \in S L(n, \mathbb{Z})$ be such that $A p_{+}=p_{-}$, let $l_{+}:\left\{v \in \mathbb{R}^{n} \mid\|v\|<.5\right\} \rightarrow \mathbb{T}^{n}$ be a linear coordinate system at $p_{+}$and $l_{-}=A \circ l_{+}$the corresponding coordinates at $p_{-}$. Next let $S=\left(\mathbb{R}^{n}-\{0\}\right) / \mathbb{R}^{\times}$ and for a vector $v \in \mathbb{R}^{n}$, denote its class in $L$ by $[v]$. Define

$$
L=\left\{([v], u) \in S \times \mathbb{R}^{n} \mid u=y v \text { for some } y \in \mathbb{R}^{\times}\right\}
$$

and let

$$
q: L \rightarrow \mathbb{R}^{n}
$$

be defined by $q([v], u)=u$. let $E \subset L$ be the set $\{([v], 0)\}$ and $L^{+}$(resp. $\left.L^{-}\right)$be the subsets $\left\{([v], u) \in S \times \mathbb{R}^{n} \mid u=y u\right.$ for some $y>0$ (resp. y $\left.\left.<0\right)\right\}$.

We will call $E$ the exceptional divisor. Note that $S L(n, \mathbb{R})$ has a natural action on $L$ and that $q$ is equivariant.

Now form a new manifold $M$. Let $V=\left\{([v], u) \in L \mid\|u\|<10^{-n}\right\}$, and let $V^{+}$(resp. $V^{-}$) be $V \cap L^{+}$(resp. $V \cap L^{-}$). Let $U=\mathbb{T}^{n}-\left\{p_{1}, p_{2}\right\}$ and form $M$ by gluing $U$ and $V$ together as follows: $l \in V^{+}$(resp $\in V^{-}$) is glued to $l_{+}(q(l))\left(\operatorname{resp} l_{-}(q(l))\right)$. Define an action of $\Gamma$ on $M$ by letting $\Gamma$ act in the standard way on $U$ and on $E \subset V$. It is immediate that this is a smooth action.

The action of $\Gamma$ on $M$ does not preserve a volume form. However, Katok and Lewis describe a modified action that does. The necessary modification is as follows: let $U$ and $V$ be as before, and let $\rho: \mathbb{R}^{n}-\{0\} \rightarrow \mathbb{R}^{n}-\{0\}$ by $\rho(u)=\|u\|^{-\alpha} u$, where $\alpha=\frac{n-1}{n}$. Form a manifold $M^{\prime}$ by gluing $\left(l \in V^{+}\right.$ (resp. $\left.V^{-}\right)$to $l^{+}(\rho(q(l)))$ (resp. $\left.l^{-}(\rho(q(l)))\right)$. Define an action of $\Gamma$ on $M^{\prime}$ by declaring it to be the standard actions on $U$ and $E \subset V$. It is not difficult to check that this action is smooth and preserves a smooth volume form.

We need expressions in local coordinates for the gluing maps from $V$ to $U$ that define $M$ and $M^{\prime}$. If $\left(X_{1}, \ldots, X_{n-1}\right) \in \mathbb{R}^{n}-0$, denote by $\left[X_{1}: \ldots X_{n}\right]$ its class in $S$ (homogeneous coordinates). Let $O \in E$; transforming by an element of $S L(n, \mathbb{R})$ if necessary, we can assume that $O=[0: \ldots: 1]$. Then define coordinates in a neighborhood of $O$ by setting

$$
\begin{gathered}
x_{i}(P)=X_{i} / X_{n}, \quad i=1, \ldots, n-1 ; \\
y(P)=q_{n}
\end{gathered}
$$

for $P=\left(\left[X_{1}: \ldots: X_{n}\right],\left(q_{1}, \ldots, q_{n}\right)\right)$; since $q_{n} X_{i}=q_{i} X_{n}$ for $i=1, \ldots, n-1$, it follows that

$$
q(P)=\left(x_{1} y, \ldots, x_{n-1} y, y\right)
$$




\section{Geometric Structures.}

If $N$ is a manifold, we denote the $k$-th order frame bundle of $N$ by $F^{k}(N)$, and by $J^{s, k}(N)$ the bundle of $k$-jets at 0 of maps from $\mathbb{R}^{s}$ to $N$. If $N$ and $N^{\prime}$ are two manifolds, and $f: N \rightarrow N^{\prime}$ is a map between them, then the $k$-jet $j^{k}(f)$ induces a map $J^{s, k} N \rightarrow J^{s, k} N^{\prime}$ for all $s$. We let $D^{k}(N)$ be the bundle whose fiber $D_{p}^{k}$ at a point $p$ consists of the set of $k$-jets at $p$ of germs of diffeomorphisms of $N$ fixing $p$. We abbreviate $D_{0}^{k}\left(\mathbb{R}^{n}\right)$ by $D_{n}^{k}$ or simply $D^{k}$; this is a real algebraic group. We will need to describe explicitly certain elements of $D^{k}$; for this purpose, observe that we can represent each element uniquely, in terms of standard coordinates $\left(\xi_{1}, \ldots, \xi_{n}\right)$ on $\mathbb{R}^{n}$, in the form

$$
\left(P_{1}\left(\xi_{1}, \ldots, \xi_{n}\right), \ldots, P_{n}\left(\xi_{1}, . ., \xi_{n}\right)\right)
$$

where $P_{1}, P_{2}, \ldots, P_{n}$ are polynomials of degree $\leq k$. We denote the vector space of such polynomial maps of degree $\leq k$ by $\mathcal{P}_{n, k}$.

We will have occasion to make calculations with these objects in local coordinates. Note that if $U \subset N$ is an open set, a choice of coordinates on $c: U^{\prime} \subset \mathbb{R}^{n} \rightarrow U$ induces a trivialization of $D^{k}(U)$ :

$$
D^{k}(U) \rightarrow U^{\prime} \times D^{k}
$$

by $j_{p}^{k}(h) \rightarrow\left(c^{-1}(p), j_{0}^{k}\left(h^{\prime}\right)\right)$ where $h^{\prime}$ is defined by: $h^{\prime}(v)=c^{-1}\left(h\left(c\left(c^{-1}(p)+\right.\right.\right.$ $v))-c^{-1}(p)$.

The group $D_{n}^{k}$ has a natural action on $F^{k}(N)$, where $n$ is the dimension of $N$. Suppose we are given an algebraic action of $D_{n}^{k}$ on a smooth algebraic variety $Z$. Then following Gromov ([12]), we make the following definition:

Definition 3.1. 1. An $A$-structure on $N$ (of order $k$, of type $Z$ ) is a smooth map $\phi: F^{k}(N) \rightarrow Z$ equivariant for the $D_{n}^{k}$ actions.

2. With notation as above, the $r$-th prolongation of $\phi$, denoted $\phi^{r}$, is the map $\phi^{r}: F^{k+r}(N) \rightarrow J^{n, r}(Z)$ defined by $\phi^{r}=j^{r}(\phi) \circ \iota_{k}^{r+k}$ where $\iota_{k}^{r+k}: F^{k+r}(N) \rightarrow J^{n, r}\left(F^{k}(N)\right)$ is the natural inclusion and $j^{k}(h):$ $J^{n, r}\left(F^{k}(N)\right) \rightarrow J^{n, r}(Z)$ is as before; this is an A-structure of type $J^{n, r}(Z)$ and order $k+r$.

Equivalently, an A-structure of type $Z$ and order $k$ is a smooth section of the associated bundle $F^{k}(N) \times_{D^{k}}(Z)$ over N. Note that an A-structure on $N$ defines by restriction an A-structure $\left.\phi\right|_{U}$ on any open set $U \subset N$. 
Remark 3.2. A-structures were introduced in [12]; a good introduction to the subject, with many examples, can be found in [4]. A comprehensive and accesible discussion of the results of [12] concerning actions of simple Lie groups can be found in [8].

Note that if $N$ and $N^{\prime}$ are $n$-manifolds, and $h: N \rightarrow N^{\prime}$ is a diffeomorphism, then $h$ induces a bundle map $j^{k}(h): F^{k}(N) \rightarrow F^{k}\left(N^{\prime}\right)$.

Definition 3.3. 1. If $\phi: F^{k}(N) \rightarrow Z, \phi^{\prime}: F^{k}\left(N^{\prime}\right) \rightarrow Z$ are A-structures, a diffeomorphism $h: N \rightarrow N^{\prime}$ is an isometry from $\phi$ to $\phi^{\prime}$ if $\phi^{\prime} \circ j^{k}(h)=$ $\phi$.

2. A local isometry of $\phi$ is a diffeomeorphism $h: U_{1} \rightarrow U_{2}$, for open sets $U_{1}, U_{2} \subset N$, which is an isometry from $\left.\phi\right|_{U_{1}}$ to $\left.\phi\right|_{U_{2}}$.

For $p \in M$ denote by $I s_{p}^{l o c}(\phi)$ the pseudogroup of local isometries of $\phi$ fixing $p$, and, for $l \geq k$, we denote by $I s_{p}^{l}(\phi)$ the set of elements $j_{p}^{l}(h) \in D_{p}^{l}$ such that $j_{p}^{l}\left(\phi \circ j_{p}^{k}(h)\right)=\phi^{l-k}$, where both sides are considered as maps $F^{k+l}(N) \rightarrow J^{l-k}(Z)$. I $s_{p}^{l}(\phi)$ is a group, and there is a natural homomorphism $r_{p}^{l ; m}: I s_{p}^{l}(\phi) \rightarrow I s_{p}^{m}(\phi)$ for $m<l$; in general, it is neither injective nor surjective.

Definition 3.4. The structure $\phi$ is called $k$-rigid if for every point $p$, the map $r_{p}^{k+1 ; k}$ is injective.

In order to provide some examples, as promised at the end of the introduction, we recall the following lemma of Gromov.

Lemma 3.5. Let $V$ be an algebraic variety and $G$ a group acting algebraically on $V$. For every $k$, there is a tautological $G$ invariant geometric structure of order $k$ on $V$, given by $\omega: P^{k}(V) \rightarrow P^{k}(V) / G$. This structure is rigid if and only if the action of $G$ on $P^{k}(V)$ is free and proper.

The conclusion in the first sentence is obvious. The second sentence is proven in section 0.4, pages 69-70, of [12].

\section{Examples}

1. The action of $G=S L_{n}(\mathbb{R})$ on $\mathbb{R}^{n}$ is algebraic. So is the action of $G$ on the manifold $N_{1}$ obtained by blowing up the origin. The reader can easily verify that the action of $G$ on $P^{2}\left(N_{1}\right)$ is free and proper. 
2. We can compactify $N_{1}$ by $N_{2}$ by viewing the complement of the blow up as a subset of the projective space $P^{n}$. Another description of the same action, which may make the rigid structure more visible to the naked eye, is as follows. $S L_{n+1}(\mathbb{R})$ acts on $P^{n}$. Let $G$ be $S L_{n}(\mathbb{R})<S L_{n+1}(\mathbb{R})$ as block diagonal matrices with blocks of size $n$ and 1 and $1 \times 1$ block equal to 1 . Then $G$ acts on $P^{n}$ fixing a point $p$. We can obtain $N_{2}$ by blowing up the fixed point $p$. The $G$ actions on both $P^{n}$ and $N_{2}$ are algebraic and again the reader can verify that the action is free and proper on $P^{2}\left(N_{2}\right)$.

3. In the construction from 2 above, there is an action of a group $H$ where $H=S L_{n}(\mathbb{R}) \ltimes \mathbb{R}^{n}$ and $G=S L_{n}(\mathbb{R})<S L_{n}(\mathbb{R}) \ltimes \mathbb{R}^{n}<S L_{n+1}(\mathbb{R})$. The $H$ action fixes the point $p$ and so also acts on $N_{2}$ algebraically. However, over the exceptional divisor, the action is never free on any frame bundle, since the subgroup $\mathbb{R}^{n}$ acts trivially to all orders at the exceptional divisor.

The behavior in example 3 above illustrates the fact that existence of rigid structures is more complicated for algebraic groups which are not semisimple, see the discussion in section 0.4.C. of [12]. This is related to our proof of the non-existence of rigid structures for the Katok-Lewis examples, which depends heavily on the fact that any such structure is locally invariant under the full affine group.

\section{An Application of Iozzi's Theorem.}

We shall use the following theorem, due to Iozzi:

Theorem 4.1. (Iozzi) Let $P$ be a principal bundle over a manifold $N$ with structure group an algebraic group $H$ and let $Y$ be an algebraic $H$ space. Let $G$ be a simple Lie group with finite center that acts on $P$ by principal bundle automorphisms, and suppose that for almost every point $p \in N$ the stabilizer of $p$ in $G$ is noncompact. Let $\Gamma \subset G$ be a lattice, and suppose that $s$ is a measurable section of the associated bundle $P \times_{H} Y$ which is essentially invariant under the action of $\Gamma$. Then it is essentially invariant under the action of $G$.

If $p \in \mathbb{T}^{n}$, denote by $S A_{p}^{\text {loc }}$ the pseudogroup of local diffeomorphisms fixing $p$ and preserving the standard connection and volume form, and by $S A_{p}^{k}$ the group of $k$-jets at $p$ of elements of $S A_{p}^{l o c}$; a choice of special affine coordinates at $p$ induces an isomorphism from $S A_{p}^{k}$ to $S L(n, \mathbb{R})$. 
Lemma 4.2. Let $\Gamma \subset S L(n, \mathbb{Z})$ be a congruence subgroup, and let $U \subset \mathbb{T}^{n}$ be a $\Gamma$ invariant open subset. Let $\phi: F^{k}(U) \rightarrow Z$ be a $\Gamma$ invariant $A$ structure. Then at each point $p \in U, S A_{p}^{\text {loc }}$ is locally contained in $I s_{p}^{\text {loc }}(\phi)$ and $S A_{p}^{k} \subset I s_{p}^{k}(\phi)$.

By "locally contained" we mean the following: if $h: U_{1} \rightarrow h\left(U_{1}\right)$ is a local diffeomorphism in $S A_{p}^{l o c}$, there is an open set $U_{1}^{\prime}, p \in U_{1}^{\prime} \subset U_{1}$, such that $\left.h\right|_{U_{1}^{\prime}} \in I s_{p}^{l o c}(\phi)$.

Proof: The second statement follows from the first. Now let $\pi: \mathbb{R}^{n} \rightarrow \mathbb{T}^{n}$ be the natural projection and let $\tilde{U}=\pi^{-1}(U)$. Then $\phi$ defines a $\Gamma$ invariant structure $\tilde{\phi}$ on $\tilde{U}$ which is also invariant by the action of $\mathbb{Z}^{n}$ by translations. Then $\tilde{\phi}$ can be thought of as a measurable section of $F^{k}\left(\mathbb{R}^{n}\right) \times{ }_{D^{k}} Z$ over $\mathbb{R}^{n}$ by ignoring the null set $\mathbb{R}^{n}-\tilde{U}$. Since the action of $S L(n, \mathbb{R})$ on $\mathbb{R}^{n}$ has non-compact stablilizers, the conditions of Iozzi's Theorem are fulfilled, and $\tilde{\phi}$ is essentially invariant under $S L(n, \mathbb{R})$. Since it is also invariant under $\mathbb{Z}^{n}$, it is invariant under the special affine group $S L(n, \mathbb{R}) \ltimes \mathbb{R}^{n}$.

Now let $p \in U$ and let $h: U_{1} \rightarrow h\left(U_{1}\right)$ be an element of $S A_{p}^{\text {loc }}$ where $U_{1}, h\left(U_{1}\right) \subset U$ are neighborhoods of $p$, and assume without loss of generality that $U_{1}$ is an evenly covered neighborhood of $p$ for the covering map $\pi$; we want to show that there is a $U_{1}^{\prime} \subset U_{1}$ such that

$$
\left.\phi \circ j^{k}(h)\right|_{U_{1}^{\prime}}=\left.\phi\right|_{U_{1}^{\prime}}
$$

$h$ lifts to a globally defined special affine transformation $\tilde{h}: \mathbb{R}^{n} \rightarrow \mathbb{R}^{n}$, and by the preceding paragraph, we know that $\tilde{\phi} \circ j^{k}(\tilde{h})=\tilde{\phi}$ almost everywhere on $\mathbb{R}^{n}$. Let $\tilde{U}_{1}$ be a connected component of $\pi^{-1}(U)$, and let $\tilde{p}$ be the point in $\tilde{U}_{1}$ such that $\pi(\tilde{p})=p$. Choose $\tilde{U}_{1}^{\prime} \subset \tilde{U}_{1}$ be such that $h\left(\tilde{U}_{1}^{\prime}\right) \subset \tilde{U}$. Then since

$$
\left.\tilde{\phi}\right|_{\tilde{U}_{1}^{\prime}} \circ j^{k}(\tilde{h})=\left.\tilde{\phi}\right|_{h\left(\tilde{U}_{1}^{\prime}\right)} \text { a.e. }
$$

and both sides are continuous sections of $F^{k} \mathbb{R}^{n} \times{ }_{D^{k}} Z$ over $h\left(\tilde{U}_{1}^{\prime}\right)$, the equality holds everywhere on $h\left(\tilde{U}_{1}^{\prime}\right)$; projecting down to $\mathbb{T}^{n}$ gives $(2)$.

\section{The Katok-Lewis Examples.}

Proposition 5.1. There is no $\Gamma$-invariant rigid A-structure on the KatokLewis example $M$.

Proof: Suppose that $\phi$ is a $\Gamma$-invariant A-structure of order $k-1$; we shall show that it cannot be rigid. In fact, we shall show that if $O$ is a point on 
the exceptional divisor $E$, there is a nontrivial element of the kernel of the $\operatorname{map} I s_{O}^{k}(\phi) \rightarrow I s_{O}^{k-1}(\phi)$.

In a neighborhood $W$ of $O$ on $E$, choose coordinates $\left(x_{1}, \ldots, x_{n-1}, y\right)$, so that in terms of these coordinates

1. $E \cap W=\{y=0\}$, and $W^{+}={ }_{\text {def }} W \cap L^{+}=\{y>0\}$;

2. the gluing map from $W-E$ to $U$ is given by

$$
\left(x_{1}, \ldots, x_{n-1}, y\right) \rightarrow\left(x_{1} y, \ldots, x_{n-1} y, y\right) ;
$$

where on $U$ we are using the coordinates $l^{+}$; call this map $\mu$. All of our constructions will be expressed in terms of these fixed coordinates.

We will define a family $\tau_{p} \in I s_{p}^{k}(\phi)$ for $p \in W^{+}$, where $\tau_{p}$ depends continuously on $p$. For $v \in \mathbb{R}^{n}$, and $b \in \mathbb{R}$, define the map

$$
U(b, v): \mathbb{R}^{n} \rightarrow \mathbb{R}^{n}
$$

by

$$
U(b, v)\left(v+\left(u_{1}, \ldots, u_{n-1}, y\right)\right)=v+\left(u_{1}+b y, u_{2}, . ., u_{n-1}, y\right) .
$$

By the lemma, we have

$$
\nu(b, p)=j_{p}^{k}\left(\mu^{-1} \circ U(b, \mu(p)) \circ \mu\right) \in I s_{p}^{k}(\phi)
$$

for any $b \in \mathbb{R}$ and $p \in W^{+}$. If we let $b: W \rightarrow \mathbb{R}$ be a continuous function, then $p \rightarrow \nu(b, p)$ defines a continuous section of $D^{k} W$.

Recall that the choice of coordinates induces a trivialization

$$
\Xi: D^{k}(W) \rightarrow W \times D^{k}
$$

write $\Xi(j)=(p, \sigma(j))$ for $j \in D^{k}(W)_{p}$. Then $\sigma(\nu(b, p))=j_{0}^{k}\left(H_{b, p}\right)$ where $H_{b, p}: \mathbb{R}^{n} \rightarrow \mathbb{R}^{n}$ is a polynomial map of degree $\leq k$ defined by

$$
\begin{gathered}
H_{b, p}\left(\xi_{1}, \ldots, \xi_{n-1}, \eta\right) \equiv_{k} \\
\left.\mu^{-1}\left(\left(x_{1}+\xi_{1}\right)(y+\eta)+b \eta,\left(x_{2}+\xi_{2}\right)(y+\eta), \ldots, y+\eta\right)\right)-\left(x_{1}, \ldots, x_{n-1}, y\right) \\
\equiv_{k}\left(\left(\xi_{1}+b \eta(y+\eta)^{-1}, \xi_{2}, \ldots, \eta\right)\right) \\
\equiv_{k}\left(\left(\xi_{1}+b \eta y^{-1}\left(1-\eta / y+(\eta / y)^{2}-\ldots+(-1)^{k-1}(\eta / y)^{k-1}\right), \xi_{2}, \ldots, \eta\right)\right)
\end{gathered}
$$

(here, we have written $f \equiv_{k} g$ for $j_{0}^{k}(f)=j_{0}^{k}(g)$ where $f$ and $g$ are germs at 0 of maps $\mathbb{R}^{n} \rightarrow \mathbb{R}^{n}$.) Now define a function $b: W \rightarrow \mathbb{R}$ by $b\left(x_{1}, \ldots, x_{n-1}, y\right)=$ $y^{k}$; then $\lim _{p \rightarrow 0} H_{b(p), p}$ exists in $\mathcal{P}_{n, k}$ and equals $H$ where

$$
H\left(\xi_{1}, . ., \xi_{n-1}, \eta\right)=\left(\xi_{1}+(-1)^{k} \eta^{k}, \xi_{2}, \ldots, \xi_{n-1}, \eta\right) .
$$


Then if

$$
\begin{gathered}
\tau_{p}=\nu(b(p), p), \\
\lim _{p \rightarrow 0} \tau_{p}=\lim _{p \rightarrow 0} \Xi^{-1}\left(p, j_{0}^{k}\left(H_{b(p), p}\right)\right)=\tau_{0}
\end{gathered}
$$

where $\tau_{0}=\Xi^{-1}\left(0, j_{0}^{k}(H)\right)$. Since $j_{0}^{k}(H)$ is in the kernel of the projection $D^{k} \rightarrow D^{k-1}, \tau_{0}$ is in the kernel of the map $D^{k}(W)_{0} \rightarrow D^{k-1}(W)_{0}$. On the other hand, since $\tau_{p} \in I s_{p}^{k}(\phi)$ for $p \in W^{+}$, by continuity $\tau_{0} \in I s_{0}^{k}(\phi)$; this violates rigidity at 0 and the proposition is proved.

Similarly, we have the following:

Proposition 5.2. There is no $\Gamma$-invariant rigid $A$-structure on $M^{\prime}$.

Proof: The argument is very similar to the one given above. Let $U, V, E$ have the same meanings as before, except that now they are to be viewed as subsets of $M^{\prime}$. Again, suppose that $\phi$ is an invariant A-structure; suppose it is rigid of order $k$. By the same argument as before, $I s_{p}^{k}(\phi)$ contains $S A_{p}^{\text {loc }}$ for all $p \in U$. Now choose a point $p$ in $E$, and choose coordinates $\left(x_{1}, \ldots, x_{n-1}, y\right)$ centered at $p$ on a neighborhood $W$ so that, with respect to these coordinates, the map $q: L \rightarrow \mathbb{R}^{n}$ has the form (1); then in terms of $l^{+}$, the gluing map from $W^{+}={ }_{\text {def }} W \cap L^{+}$to $U$ is

$$
\left(x_{1}, \ldots, x_{n-1}, y\right) \rightarrow\left(x_{1}^{2} y^{2}+\ldots+x_{n-1}^{2} y^{2}+y^{2}\right)^{-\alpha / 2}\left(x_{1} y, x_{2} y, \ldots, x_{n-1} y, y\right) .
$$

Now define new coordinates $\left(x_{1}^{\prime}, \ldots, x_{n-1}^{\prime}, y^{\prime}\right)$ on $W$ by

$$
\begin{gathered}
x_{i}^{\prime}=x_{i}, \quad i=1, \ldots, n-1 ; \\
y^{\prime}=y\left(1+x_{1}^{2}+\ldots+x_{n-1}^{2}\right)^{-\alpha /(2-2 \alpha)}=y\left(1+x_{1}^{2}+\ldots+x_{n-1}^{2}\right)^{(1-n) / 2} .
\end{gathered}
$$

Then in terms of these coordinates, the map (3) becomes

$$
\left(x_{1}^{\prime}, \ldots, x_{n-1}^{\prime}, y^{\prime}\right) \rightarrow\left(x_{1}^{\prime} y^{\prime \delta}, \ldots, x_{n-1}^{\prime} y^{\prime} \delta, y^{\prime \delta}\right)
$$

where $\delta=1-\alpha=\frac{1}{n}$. Call this map $\mu^{\prime}$. Let $U(b, v)$ have the same meaning as in the previous proposition; again, we conclude that

$$
\nu^{\prime}(p, b)=j_{p}^{k}\left(\mu^{\prime-1} \circ U\left(b, \mu^{\prime}(p)\right) \circ \mu^{\prime}\right)
$$

is in $I s_{p}^{k}(\phi)$. Using the trivialization

$$
\Xi^{\prime}: D^{k}(W) \rightarrow W \times D^{k}
$$


given by this choice of coordinates, we have that $\nu^{\prime}(p, b)=\Xi^{\prime-1}\left(p, j_{0}^{k}\left(H_{b, p}^{\prime}\right)\right)$ for a polynomial map $H_{b, p}^{\prime}: \mathbb{R}^{n} \rightarrow \mathbb{R}^{n}$ defined by:

$$
\begin{gathered}
H_{b, p}^{\prime}\left(\xi_{1}^{\prime}, \ldots, \xi_{n-1}^{\prime}, \eta^{\prime}\right) \equiv_{k} \\
\mu^{\prime-1}\left(\left(x_{1}^{\prime}+\xi_{1}^{\prime}\right)\left(y^{\prime}+\eta^{\prime}\right)^{\delta}+b\left(\left(y^{\prime}+\eta^{\prime}\right)^{\delta}-y^{\prime}\right),\left(x_{2}^{\prime}+\xi_{2}^{\prime}\right)\left(y^{\prime}+\eta^{\prime}\right)^{\delta}, \ldots\right. \\
\left.\ldots,\left(x_{n-1}^{\prime}+\xi_{n-1}^{\prime}\right)\left(y^{\prime}+\eta^{\prime}\right)^{\delta},\left(y^{\prime}+\eta^{\prime}\right)^{\delta}\right)-\left(x_{1}^{\prime}, \ldots, x_{n-1}^{\prime}, y^{\prime}\right) \\
\equiv_{k}\left(\xi_{1}^{\prime}+b\left(1-y^{\prime} \delta\left(y^{\prime}+\eta^{\prime}\right)^{-\delta}\right), x_{2}^{\prime}, \ldots, \xi_{n-1}^{\prime}, \eta^{\prime}\right) \\
\equiv_{k}\left(\xi_{1}^{\prime}+b\left(1-\left(1+\eta^{\prime} / y^{\prime}\right)^{-\delta}\right), x_{2}^{\prime}, \ldots, \xi_{n-1}^{\prime}, \eta^{\prime}\right) \\
\equiv_{k}\left(\xi_{1}^{\prime}+b\left(-r_{1} \eta^{\prime} / y^{\prime}-r_{2}\left(\eta^{\prime} / y^{\prime}\right)^{2}-\ldots-r_{k}\left(\eta^{\prime} / y^{\prime}\right)^{k}\right), x_{2}^{\prime}, \ldots, \xi_{n-1}^{\prime}, \eta^{\prime}\right)
\end{gathered}
$$

where $1+r_{1} X+r_{2} X^{2}+\ldots$ is the Taylor series of $(1+X)^{-\delta}$. Then if we let $b(p)=y^{\prime k}$ as before, we have

$$
\lim _{p \rightarrow 0} \nu^{\prime}(p, b(p))=\tau_{0}^{\prime}
$$

where

$$
\tau_{0}^{\prime}\left(\xi_{1}^{\prime}, \ldots, \xi_{n-1}^{\prime}, \eta^{\prime}\right)=\left(\xi_{1}^{\prime}+r_{k} \eta^{\prime k}, \xi_{2}^{\prime}, \ldots, \xi_{n-1}^{\prime}, \eta^{\prime}\right) .
$$

Again, continuity implies that $\tau_{0}^{\prime} \in I s_{0}^{k}(\phi)$, which violates the $k$-rigidity of $\phi$ at 0 .

\section{G actions.}

In this section we will show how to adapt the results of section 5 to the setting of $\mathrm{G}$ actions. Here we will be considering examples where $G$ acts on $H / \Lambda$ and we blow up and glue along two closed $G$ orbits.

The proof follows the same outline as before. First we use Iozzi's theorem to construct many local vector fields that preserve the structure. Second, we show that these vector fields define infinitesimal isometries that degenerate on the exceptional divisor.

We first describe the adaptations necessary to use Theorem 4.1. Here our model space is $H / \Lambda$ rather than $\mathbb{T}^{n}$. Given any point $p$ of $H$, we can define an $H$ action fixing $p$ by translating by $p^{-1}$, acting by $A d(h)$ and then translating back. This defines a pseudogroup of local diffeomorphisms near any point $p$ of $H / \Lambda$, which we denote by $H_{p}^{l o c}$. We can clearly restrict this action to any subgroup $G<H$, and we denote the corresponding pseudogroup by $G_{p}^{l o c}$. The version of Lemma 4.2. that we need is: 
Lemma 6.1. Let $G$ and $H$ be simple real algebraic Lie groups, $G<H$. Let $\Lambda$ be a lattice in $H$, so $G$ acts on $H / \Lambda$. Let $U \subset H / \Lambda$ be an open dense $G$ invariant set. Let $\phi: F^{k}(U) \rightarrow Z$ be a $G$ invariant $A$-structure. Then at each point $p \in U, G_{p}^{\text {loc }}$ is locally contained in $I s_{p}^{\text {loc }}(\phi)$.

Proof. We proceed as in the proof of Lemma 4.2. Let $\pi: H \rightarrow H / \Lambda$ be the natural projection and let $\tilde{U}=\pi^{-1}(U)$. Now $\phi$ defines a geometric structure $\tilde{\phi}: \tilde{U} \rightarrow Z$ which is also right $\Lambda$ invariant. We can think of $\phi$ as a measurable section of $F^{k}(H) \times{ }_{D^{k}} Z$. Here the $\Lambda$ action does not have noncompact stabilizers, so we need an additional trick. Since $\phi$ is $G$ invariant, we can think of $\tilde{\phi}$ as a $\Lambda$ invariant measurable section of the $Z$ bundle over $G \backslash H$ given by $G \backslash F^{k}(H) \times_{D^{k}} Z$. Since $G \backslash F^{k}(H) \rightarrow G \backslash H$ is a $D^{k}$ bundle, and the stabilizers of points for the $H$ action on $G \backslash H$ are all non-compact, we are in the setting where Iozzi's theorem applies. This implies that $\tilde{\phi}$ is invariant under the right $H$ action on $H$. The rest of the proof of the lemma proceeds exactly as in Lemma 4.1.

Now let $M$ be $H / \Lambda$ with a closed $G$ orbit blown-up as in [2]. Let $M^{\prime}$ be the modification of $M$ on which there is a $G$ invariant volume form as described in [2]. (The modification here is analogous to the one described in section 2 for the Katok-Lewis examples and is described in detail in [2].) We have the following:

Proposition 6.2. There is no $G$ invariant rigid $A$-structure on either $M$ or $M^{\prime}$.

In order to prove the proposition we must describe local coordinates on $M$ and $M^{\prime}$ as in section 2 . In fact, we will only prove the proposition for $M$ since the modifications necessary for $M^{\prime}$ are exactly as in section 5 .

We outline some of the construction of $M$ from section 2 of [2] in order to describe the local coordinates in which we will carry out our computation. Let $M_{0}=H / \Lambda$. We can identify $T M_{0}$ with $M_{0} \times \mathfrak{h}$. The derivative action is given by $h(m, v)=(h m, A d(h) v)$. The tangent bundle to the $G$ orbits is clearly isomorphic to $M_{0} \times \mathfrak{g}$. Since $G$ is semisimple, there is a $G$ invariant complement to $\mathfrak{g}$ in $\mathfrak{h}$, which we will call $V$. Now $M_{0} \times V$ is a $G$ invariant sub-bundle of $T M_{0}$. Given a closed $G$ orbit $N$ in $M_{0}$, we can find a tubular neighborhood $U$ of $N$ and a neighborhood $U^{\prime}$ in $V$ such that the natural map $\exp _{V}: N \times U^{\prime} \rightarrow U$ is a diffeomorphism. We construct $M$ by blowing up along two closed $G$ orbits $N_{1}$ and $N_{2}$ and gluing. To do this we work in a 
tubular neighborhood of each $N_{i}$. Here we merely sketch the construction locally, the reader is referred to [2] for details.

Let $S=\left(\mathbb{R}^{m}-0\right) / \mathbb{R}^{\times}$. We take a subset of $\mathbb{R}^{n} \times \mathbb{R}^{m} \times S$ of the form

$$
L=\left\{(x, v,[u]) \in \mathbb{R}^{n} \times \mathbb{R}^{m} \times S \mid v=c u \text { for some } c \neq 0\right\}
$$

and let $q: L^{+} \rightarrow \mathbb{R}^{n+m}$ be the canonical projection. Mimicking the construction in section 2 , we also introduce similar coordinates in a neighborhood of $N_{2} \times U_{0}$. As before, we partition $L$ into three sets:

$$
\begin{aligned}
& L^{+}=\left\{(x, v,[u]) \in \mathbb{R}^{n} \times \mathbb{R}^{m} \times S \mid v=c u \text { for some } c>0\right\} \\
& L^{-}=\left\{(x, v,[u]) \in \mathbb{R}^{n} \times \mathbb{R}^{m} \times S \mid v=c u \text { for some } c<0\right\}
\end{aligned}
$$

and $E=\{\vec{x}, 0,[u])\}$.

Introduce local coordinates $l^{+}$on a neighborhood $N_{1} \times U_{0}$ of the form $\left(v_{1}, \ldots, v_{m}, x_{1} \ldots x_{n}\right)$ where the $x_{i}$ are coordinate along $U_{0}$ directions and the $v_{j}$ are coordinates on $N_{1}$. Mimicking the construction in section 2, we also introduce similar coordinates $l^{-}$in a neighborhood of $N_{2} \times U_{0}$. We form $M$ by identifying taking $\left(M_{0}-N_{1} \cup N_{2}\right) \cup L / R$ where $R$ is the relation defined by identifying $q\left(L^{+}\right)$with $l^{+}\left(N_{1} \times U_{0}-N_{1}\right)$ and similarly for $q\left(L^{-}\right)$with $l^{-}\left(N_{2} \times U_{0}-N_{2}\right)$.

We are being somewhat careless here. The construction we are outlining does "blow-up" along a subspace in a Euclidean space. Since $N_{i}$ are not contractible the maps $l^{+}$and $l^{-}$above are not well-defined. To make sure that everything is canonically defined in a neighorhood of $N_{i}$ in $M_{0}$ we should define $L$ intrinsically. This is done in section 2 of [2] and we refer the reader there for the necessary justifications. Since we are only interested in a computation done in the neighborhood of a point in $E$, the construction above is sufficient for our purposes.

Proof. We follow the proof of Proposition 5.1. The main difficulty is to replace the map $U(b, v)$ used there by a local isometry on $H / \Lambda$ for which the computation does not become intractable. As in that proposition, assume there is a $G$ invariant $A$-structure $\phi$ of order $k-1$. Once again, we will show that if $O$ is a point on the exceptional divisor $E$, there is a non-trivial element in the kernel of the map $I s_{O}^{k}(\phi) \rightarrow I s_{O}^{k-1}(\phi)$.

In order to define the infinitesimal isometry and complete the computation here, we must chose our coordinates and the vector field defining the infinitesimal isometry carefully. We will use facts about the structure of simple Lie groups and their Lie algebras, using [15] as a reference. We first 
pick a Cartan subalgebra $\mathfrak{a}<\mathfrak{h}$, and then note that $\mathfrak{h}=\mathfrak{a} \oplus \bigoplus_{\alpha} X_{\alpha}$ where $X_{\alpha}$ is a root subspace for a root $\alpha$ and $\alpha$ runs over all roots. We pick $\mathfrak{a}$ such that $\mathfrak{a} \cap \mathfrak{g}$ is a Cartan subalgebra of $\mathfrak{g}$. We chose coordinates on $H$ that are obtained by exponentiating these coordinates so as to be able to use the Baker-Campbell-Hausdorff formula to describe multiplication. We choose a vector field $V \in \mathfrak{g}$ such that $V=X_{\alpha}$ for a simple root $\alpha$. It follows that $\left[V, X_{\beta}\right] \in X_{\alpha+\beta}$ for any root $\beta$ and that $[V, W]=\alpha(W) V$ for $W \in \mathfrak{a}$. We pick a vector $Y \in \mathfrak{h}$ such that $Y \notin \mathfrak{g}$ and such that $Y=X_{\beta}$ where $\beta$ is a simple root and $X_{\alpha+\beta} \neq \emptyset$. Such a $Y$ exists, since otherwise either (1) all simple root spaces for $\mathfrak{h}$ are in $\mathfrak{g}$ forcing $\mathfrak{g}=\mathfrak{h}$ or $(2) \mathfrak{g}$ commutes with it's complement in $\mathfrak{h}$, in which case, $\mathfrak{h}$ cannot be simple. We further note that $X_{\alpha+\beta}$ is not in $\mathfrak{g}$. Otherwise $X_{\beta}=\left[X_{-\alpha}, X_{\beta+\alpha}\right]$ would also be in $\mathfrak{g}$. (For discussion of structure theory that makes all of this transparent, see section 4.3 of [15].)

Letting $b$ be a real number, we observe that $\operatorname{Ad}(\exp (b V)(\exp (M))=$ $\exp \left(M+[b V, M]+b^{2} A(V, M)\right)$ for any $M$ in a small neighborhood of 0 in $\mathfrak{h}$, where $A(X, M)$ is a sum of various higher order brackets of $M$ with $V$ with coeffecients powers of $b$. This follows from the Baker-Campbell-Hausdorff formula, see e.g. section 2.15 of [15].

We now choose our coordinates $\left(v_{1}, \ldots, v_{m}, x_{1}, \ldots, x_{n-1}, y\right)$ in a neighborhood $W$ of 0 subject to the following specifications. The coordinates are given by exponentiating a basis for $\mathfrak{h}$ consistent with the decomposition $\mathfrak{h}=\mathfrak{a} \oplus \bigoplus_{\alpha} X_{\alpha}$. We will let $y$ be the coordinate corresponding to $\exp (t Y), v_{1}$ the coordinate corresponding to $\exp (t V)$, and $x_{1}$ the coordinate corresponding to $\exp (t[V, Y])$ which is nontrivial by the considerations in the preceding paragraph. We also assume that the $v_{1}$ coordinate corresponding to $\exp (V)$ is one.

Furthermore we choose the coordinates $\left(v_{1}, \ldots v_{m}, x_{1}, \ldots, x_{n-1}, y\right)$, so that in terms of these coordinates

1. $E \cap W=\left\{x_{1}=\cdots=x_{m}=y=0\right\}$, and $W^{+}={ }_{\text {def }} W \cap L^{+}=\{y>0\}$;

2. the gluing map from $W-E$ to $U$ is given by

$$
\left(v_{1}, \ldots, v_{m}, x_{1}, \ldots, x_{n-1}, y\right) \rightarrow\left(v_{1}, \ldots, v_{m}, x_{1} y, \ldots, x_{n-1} y, y\right)
$$

where on $U$ we are using the coordinates $l^{+}$; call this map $\mu$. All of our constructions will be expressed in terms of these fixed coordinates.

We will define a family $\tau_{p} \in I s_{p}^{k}(\phi)$ for $p \in W^{+}$, where $\tau_{p}$ depends continuously on $p$. Let $w \in \mathbb{R}^{m+n}=\mathfrak{h}$ and $V \in \mathfrak{g}$ be as above, and $b \in \mathbb{R}$, define the map

$$
U(b V, w): \mathfrak{h} \rightarrow \mathfrak{h}
$$


by

$$
\begin{gathered}
U(b V, w)\left(w+\left(v_{1}, \ldots v_{m}, u_{1}, \ldots, u_{n-1}, y\right)\right)= \\
w+\operatorname{Ad}(\exp (b V))\left(v_{1}, \ldots, v_{m}, u_{1}, u_{2}, . ., u_{n-1}, y\right) .
\end{gathered}
$$

By lemma 6.1, we have

$$
\nu(b V, p)=j_{p}^{k}\left(\mu^{-1} \circ U(b V, \mu(p)) \circ \mu\right) \in I s_{p}^{k}(\phi)
$$

for any $b \in \mathbb{R}$ and $p \in W^{+}$. If we let $b: W \rightarrow \mathbb{R}$ be a continuous function, then $p \rightarrow \nu(b, p)$ defines a continuous section of $D^{k} W$.

Recall that the choice of coordinates induces a trivialization

$$
\Xi: D^{k}(W) \rightarrow W \times D^{k}
$$

write $\Xi(j)=(p, \sigma(j))$ for $j \in D^{k}(W)_{p}$. Then $\sigma\left(\nu(b V, p)=j_{0}^{k}\left(H_{b V, p}\right)\right.$ where $H_{b V, p}: \mathbb{R}^{m+n} \rightarrow \mathbb{R}^{m+n}$ is a polynomial map of degree $\leq k$ defined by

$$
\begin{gathered}
H_{b V, p}\left(\alpha_{1}, \ldots \alpha_{m}, \xi_{1}, \ldots, \xi_{n-1}, \eta\right) \equiv_{k} \\
\mu^{-1}\left(U(b V, \mu(p))\left(v_{1}+\alpha_{1}, \ldots, v_{m}+\alpha_{m},\left(x_{1}+\xi_{1}\right)(y+\eta), \ldots, y+\eta\right)\right) \\
-\left(v_{1}, \ldots, v_{m}, x_{1}, \ldots, x_{n-1}, y\right) \equiv_{k} \\
\left.\mu^{-1}\left(v_{1}+\alpha_{1}+q_{1}(b), \ldots, v_{m}+\alpha_{m}+q_{n}(b),\left(x_{1}+\xi_{1}\right)(y+\eta)+p_{1}(b), \ldots, y+\eta\right)\right) \\
-\left(v_{1}, \ldots, v_{m}, x_{1}, \ldots, x_{n-1}, y\right) \equiv_{k} \\
\left(\left(\alpha_{1}+q_{1}(b), \ldots, \alpha_{m}+q_{m}(b), \xi_{1}+p_{1}(b)(y+\eta)^{-1}, \xi_{2}+p_{2}(b)(y+\eta)^{-1}, \ldots, \eta\right)\right.
\end{gathered}
$$

Here each $q_{i}$ is a polynomial in $b$ with zero constant term and linear term either zero or $\alpha_{j(i)} b$ for some $j(i) \in\{1, \ldots m\}$. Each $p_{l}$ for $l \neq 1$ is a polynomial in $b$ with zero constant term and linear term either zero or $\left(x_{k(l)} \eta+\xi_{k(l)}(y+\right.$ $\eta)) b$ for $k(l) \in\{1, \ldots, n-1\}$. The remaining polynomial $p_{1}$ has zero constant term and has linear term $\eta b$. This follows by direct computation using the Baker-Campbell-Hausdorff formula as discussed above, the formula for $[V, M]$ deduced from the fact that $V$ is a root space for a simple root for $\mathfrak{g}$ and the fact that $Y$ is a root space for a simple root for $\mathfrak{h}$. We note here that any of the polynomials $q_{i}, p_{j}$ with trivial linear term is trivial, though we do not need this for our computation.

Let $p^{\prime}$ be a point on the singular divisor, i.e, a point where $x_{1}=\cdots=$ $x_{m}=y=0$. Now define a function $b: W \rightarrow \mathbb{R}$ by

$$
b\left(v_{1}, \ldots v_{m}, x_{1}, \ldots, x_{n-1}, y\right)=y^{k},
$$


make the substitution $(y+\eta)^{-1} \equiv_{k}\left(1-\eta / y+(\eta / y)^{2}-\ldots+(-1)^{k}(\eta / y)^{k}\right)$ and compute as in the proof of Proposition 5.1. Then $\lim _{p \rightarrow p^{\prime}} H_{b(p) V, p}$ exists in $\mathcal{P}_{n, k}$ and equals $H$ where

$$
H\left(\alpha_{1}, \ldots, \alpha_{m}, \xi_{1}, . ., \xi_{n-1}, \eta\right)=\left(\alpha_{1}, \ldots, \alpha_{m}, \xi_{1}+(-1)^{k} \eta^{k}, \xi_{2}, \ldots, \xi_{n-1}, \eta\right) .
$$

Then if

$$
\begin{gathered}
\tau_{p}=\nu(b(p), p), \\
\lim _{p \rightarrow p^{\prime}} \tau_{p}=\lim _{p \rightarrow p^{\prime}} \Xi^{-1}\left(p, j_{0}^{k}\left(H_{b(p), p}\right)\right)=\tau_{p^{\prime}}
\end{gathered}
$$

where $\tau_{p^{\prime}}=\Xi^{-1}\left(0, j_{0}^{k}(H)\right)$. Since $j_{0}^{k}(H)$ is in the kernel of the projection $D^{k} \rightarrow D^{k-1}, \tau_{p^{\prime}}$ is in the kernel of the map $D^{k}(W)_{0} \rightarrow D^{k-1}(W)_{0}$. On the other hand, since $\tau_{p} \in I s_{p}^{k}(\phi)$ for $p \in W^{+}$, by continuity $\tau_{p^{\prime}} \in I s_{p^{\prime}}^{k}(\phi)$; this violates rigidity at $p^{\prime}$ and the proposition is proved.

\section{Almost rigid structures.}

In this section, we define a generalization of rigid A-structures and show that it is possible to construct such an object on the Katok-Lewis examples. We continue to use the notation of section 2 .

Definition 7.1. An A-structure $\phi$ is called $(j, k)$-almost rigid (or just almost rigid) if for every point $p, r_{p}^{k, k-1}$ is injective on the subgroup $r^{k+j, k}\left(I s^{k+j}\right) \subset I s^{k}$.

Thus $k$-rigid structures are the $(0, k)$-almost rigid structures.

Basic Example: Let $V$ be an $n$-dimensional manifold. Let $X_{1}, \ldots X_{n}$ be a collection of vector fields on $M$. This defines an A-structure $\psi$ of type $\mathbb{R}^{n^{2}}$ on $M$. If $X_{1}, \ldots, X_{n}$ span the tangent space of $V$ at every point, then the structure is rigid in the sense of Gromov. Suppose instead that there exists a point $p$ in $V$ and $X_{1} \wedge \ldots \wedge X_{n}$ vanishes to order $\leq j$ at $p$ in $V$. Then $\psi$ is a $(j, 1)$-almost rigid structure. Indeed, let $p \in M$, and let $\left(x_{1}, \ldots, x_{n}\right)$ be coordinates around $p$. Suppose that in terms of these coordinates, $X_{l}=a_{l}^{m} \frac{\partial}{\partial x_{j}}$. Suppose that $f \in I s_{p}^{j+1}$. We must show that $r_{p}^{j+1,1}(f)$ is trivial. Let $\left(f^{1}, \ldots, f^{n}\right)$ be the coordinste functions of $f$. Then $f \in I s_{p}^{j+1}$ implies that

$$
a_{k}^{l}-a_{k}^{m} \frac{\partial f^{l}}{\partial x^{m}}
$$

vanishes to order $j+1$ at $p$ for all $k$ and $l$. Let $\left(b_{k}^{l}\right)$ be the matrix so that $b_{k}^{m} a_{m}^{l}=\operatorname{det}\left(a_{r}^{s}\right) \delta_{k}^{l}$. Multiplying expression (7.1) by $\left(b_{k}^{l}\right)$, we see that 
$\operatorname{det}\left(a_{r}^{s}\right)\left(\delta_{k}^{l}-\frac{\partial f^{l}}{\partial x^{k}}\right)$ vanishes to order $j+1$. But since by assumption $\operatorname{det}\left(a_{r}^{s}\right)$ vanishes to order $\leq j$, this implies that $\left(\partial f^{l} / \partial x^{k}\right)(p)=\delta_{k}^{l}$, so $r_{p}^{j+1,1}(f)$ is the identity, as required.

If confused by the notation, the interested reader may find it enlightening to work out the basic example in the trivial case $n=1$. Similar arguments can be given to show that frames that degenerate to subframes are also almost rigid, provided the order of vanishing of the form defining the frame is always finite.

Here we will show that the Katok-Lewis examples support an invariant $(1,2)$-almost rigid structure.

Observe that $G L(n, \mathbb{R})$ acts on $J^{2}\left(\mathbb{R}^{n}, \mathbb{R}^{n}, 0,0\right)$ by composition on the right. Let $U \subset J^{2}\left(\mathbb{R}^{n}, \mathbb{R}^{n}, 0,0\right)$ be the open subvariety of points where the action is free, and let $Z$ be the quotient. Note that $D^{k}$ acts on $V$ by composition on the left. Given $x$ in $\mathbb{R}^{n}$, we denote by $T_{x}$ translation by $x$.

Definition 7.2. The canonical generalized connection on $L$ is the equivariant map $\Phi: F^{2}(L) \rightarrow Z$ defined as follows: if $j \in F^{2}(L)_{z}$ let $f: W \subset$ $\mathbb{R}^{n} \rightarrow L$ be a map such that $j_{0}^{2}(f)=j$. Then $\Phi(j)$ is the class in $V$ of $j_{0}^{2}\left(T_{q(f(p))} \circ q \circ f\right) \in J^{2}\left(\mathbb{R}^{n}, \mathbb{R}^{n}, 0,0\right)$.

To justify this definition, we need to check that $j_{0}^{2}\left(T_{q(f(p))} \circ q \circ f\right)$ is in the set $U$. This is clear if $p$ is not in the exceptional divisor, for then this is the jet of a diffeomorphism. Now suppose $p$ is in the exceptional divisor; we can assume that $p=([0: 0: \cdots: 0: 1],(0, \cdots, 0))$. Then we can choose coordinates $\mu: \mathbb{R}^{n} \rightarrow L$ near $p$ as usual by $\mu\left(x_{1}, \cdots, x_{n-1}, y\right)=\left(\left[x_{1}\right.\right.$ : $\left.\left.\cdots: x_{n-1}: 1\right],\left(x_{1} y, \cdots x_{n-1} y, y\right)\right)$ and the map $q \circ \mu$ is $\left(x_{1}, \cdots, x_{n-1}, y\right) \rightarrow$ $\left(x_{1} y, \cdots x_{n-1} y, y\right)$ and it is easy to check that the stabilizer of the 2 -jet of this map for the right action of $G L(n, \mathbb{R})$ is trivial.

Proposition 7.3. The above construction defines a smooth (1,2)-almost rigid A-structure, invariant for the $S L(n, \mathbb{R})$ action on $L$.

Remark 7.4. The structure $\Phi$ is the "same as" the standard connection on $\mathbb{R}^{n}$ on the complement of the exceptional divisor in the following sense: The diffeomorphism $\pi: L-E \rightarrow \mathbb{R}^{n}-0$ allows one to pull back the standard connection to a connection on $L-E$, which is the same as an equivariant map

$$
\Psi:\left.F^{2}(L)\right|_{L-E} \rightarrow D^{2}(n, \mathbb{R}) / G L(n, \mathbb{R}) \subset V ;
$$

this map coincides with the restriction of $\Phi$ to $\left.F^{2}\right|_{L-E}$. 
In particular, if $h: U \subset L-E \rightarrow U^{\prime} \subset L-E$ is an affine map, it is also an isometry of the $Z$ - structure.

This construction allows one to put an almost rigid structure on the manifold $M$, as follows. Recall that $M$ can be written as the union of a subset $U \subset L$ and a subset $U^{\prime} \subset \mathbb{T}^{n}$, where the gluing map $h: U-(U \cap E) \rightarrow U^{\prime}$ is affine. Let $\Phi$ be the restriction of the canonical generalized connection on $L$ to $U$. Remark 7.4 shows that a connection defines a structure $\Psi$ of type $Z$ on $U^{\prime}$, and that $h$ is an isometry of $Z$-structures. Thus $\Phi$ and $\Psi$ paste together to form structure of type $Z$ on $M$. Let us call this structure $\phi$; the construction shows that it is invariant under the $\Gamma$ action on $M$. Now:

Proposition 7.5. The structure $\phi$ is (1,2)-almost rigid.

Proof: We need to show that for every point $p$ in $M$,

$$
r_{p}^{2,1}: r^{3,2}\left(I s_{p}^{3}(\phi)\right) \rightarrow I s^{1}(\phi)
$$

is injective. This is clear for $p$ not in $E$, since there $\phi$ defines a connection, which is rigid in the usual sense. Now if $p \in E$, we may assume without loss of generality that $p=([0: 0: \cdots: 0: 1],(0, \cdots, 0)$ and that coordinates $\left(x_{1}, \ldots, x_{n-1}, y\right)$ are chosen near $p$ so that $q$ is expressed in these coordinates as $\left(x_{1}, \ldots, x_{n-1}, y\right) \rightarrow\left(x_{1} y, \ldots, x_{n-1} y, y\right)$. Now let $F=\left(F^{1}, \ldots, F^{n}\right) \in$ $I s_{p}^{3}(\phi) ;$ then

$$
\begin{aligned}
F^{1}\left(x_{1}, \ldots x_{n-1}, y\right) F^{n}\left(x_{1}, \ldots, x_{n-1}, y\right) & \equiv_{3} x_{1} y \\
F^{2}\left(x_{1}, \ldots x_{n-1}, y\right) F^{n}\left(x_{1}, \ldots, x_{n-1}, y\right) & \equiv_{3} x_{2} y \\
& \vdots \\
F^{n}\left(x_{1}, \ldots, x_{n-1}, y\right) & \equiv_{3} y .
\end{aligned}
$$

Now assume that $r^{3,1}(F)$ is trivial; then

$$
F^{i}\left(x_{1}, \ldots x_{n-1}, y\right)=x^{i}+P^{i}\left(x_{1}, \ldots, x_{n-1}, y\right)
$$

for $i=1, \ldots, n$, where the $P^{i}$ vanish to order 1 at 0 . Inserting these expressions into (7.2), we see first, that $P^{n}$ vanishes to order 3 at 0 ; then that $x_{i} P^{n}+y P^{i}+P^{i} P^{n}$ vanishes to order 3 at 0 ; but since $P^{n}$ vanishes to order 3 , this implies that $y P^{i}$ vanishes to order 3 , so that $P^{i}$ vanishes to order 2 . This completes the proof. 


\section{Gromov's theorem for almost rigid structures and other questions.}

One of the principal results of [12] is:

Theorem 8.1. Let $G$ be a simple Lie group and suppose that $G$ acts analytically on a compact manifold $M$ preserving a volume and an analytic rigid geometric structure. Further assume the action is ergodic. Then there exists a linear representation $\sigma: \pi_{1}(M) \rightarrow G L_{n}(\mathbb{R})$ such that the Zariski closure of $\sigma\left(\pi_{1}(M)\right)$ contains a group locally isomorphic to $G$.

At this juncture the question arises as to whether Gromov's theorem remains true for almost rigid structures or at least for the particular structure described above on the Katok-Lewis example. A careful examination of Gromov's proof shows that he only uses the rigid structure twice. Once to show that $M$ is locally homogeneous on an open dense set and once, in conjunction with analyticity, to show that local isometries extend uniquely to global ones on simply connected manifolds. The key step in proving that the manifold is locally homogeneous on an open dense set is showing that infinitesimal isometries extend to local ones. Gromov's proof of this fact still works for almost rigid $A$-structures on $M$ that are rigid on an open dense subset $U$ of $M$. Since all the examples above of almost rigid structures are rigid on an open dense set, this leads to the natural:

Question 1. Is a $(j, k)$-almost rigid structure on a manifold $M$ always $k$ rigid on an open dense set? Is this true if we further assume there is a group action preserving the structure which has a dense orbit?

We believe the answer to this question is positive, even without the additional assumption. The remaining obstacle to proving Gromov's theorem is surprisingly simple. He proves that a locally defined analytic Killing field $V$ of an analytic rigid $A$-structure on a simply connected manifold $X$ has a unique analytic continuation. This is an easy generalization of a similar theorem for $G$-structures of finite type by Amores [1]. To complete the proof of Gromov's theorem for almost rigid structures, one only needs to prove an analogue of Amores' theorem for almost rigid $A$-structures. However, here one needs to be more careful.

If we let $M$ be the modified Katok-Lewis example from above, we can show that no representation exists for $N=(G \times M) / \Gamma$. The proof is extremely indirect, and we merely sketch it here. The fundamental group for $M$ is an $H N N$ extension of $\mathbb{Z}^{n} * \mathbb{Z}_{2} * \mathbb{Z}_{2}$ where the automorphism $T$ defining the $H N N$ extension switches the $\mathbb{Z}_{2}$ 's. The fundamental group of $N$ is 
$\Gamma \ltimes \pi_{1}(M)$ where $\Gamma<S L_{n}(\mathbb{Z})$ is a subgroup of finite index. Note that $N$ is non-compact. However, this is not an obstacle to a proof of Gromov's theorem and this exact difficulty is dealt with in [11]. In that paper, it is also shown that if Gromov's representation $\sigma$ exists for $N$, it restricts to a non-trivial representation $\sigma$ of $\pi_{1}(M)$. Using the explicit construction of Gromov's representation, the fact that the rigid structure on $M$ is the standard connection on $\mathbb{T}^{n}$ off the exceptional divisor $E$ and the analysis contained in [11], one can show that $\sigma\left(\pi_{1}(M)\right)$ surjects onto $\mathbb{Z}^{n}$. Furthermore, one can see that this surjection and $\sigma$ are both $\Gamma$-equivariant. The $\Gamma$ action on $\pi_{1}(M)$ comes from the inclusion $\Gamma<\operatorname{Aut}\left(\pi_{1}(M)\right)=S L_{n}(\mathbb{Z})$ If we now apply the main theorem of [10], we see that this implies that there is a $\Gamma$ equivariant map $\phi$ from $M$ to $\mathbb{T}^{n}$ inducing the surjection on fundamental groups. It is easy to see that this is impossible by showing that $\phi$ is the identity off the exceptional divisor and then considering the possibilities for $\phi(E)$.

The argument of the preceding paragraphs is clearly unsatisfactory as a proof that analytic continuation of vector fields is not always possible for almost rigid $A$-structures. We note here that the above argument fails to produce a contradiction if one simply passes to an appropriate double cover of $M$ before beginning the construction. It would be interesting to determine a natural additional condition on an almost rigid structure that would allow one to prove an analogue of Amores' result.

\section{References.}

[1] Amores, A. M., Vector fields of a finite type G-structure, J. Diff. Geom. 14 (1979) 1-6.

[2] Benveniste, E.J. Exotic actions of semisimple groups and their deformations. to appear in Israel J. Math.

[3] Benoist, Y., Foulon, P., and Labourie, F., Flots d'Anosov a distibutions stable et instable differentiables, JAMS 5 (1992)33-74.

[4] Benoist, Y. Orbites de structures rigides. Integrable systems and foliations (Montpellier 1995) Boston: Birkhauser, 1997

[5] Candel, A.; Quiroga-Barranco, R. Gromov's centralizer theorem. Geom. Dedicata 100 (2003), 123-155.

[6] Candel, A.; Quiroga-Barranco, R. Parallelisms, prolongations of Lie algebras and rigid geometric structures, preprint. 
[7] Candel, A.; Quiroga-Barranco, R. Rigid and finite type geometric structures, preprint.

[8] Feres, R. Rigid geometric structures and actions of semisimple Lie groups. Rigidité, groupe fondamental et dynamique, 169-188, Panor. Synthèses, 13, Soc. Math. France, Paris, 2002.

[9] Feres, R. Rigid transformation groups and local normal forms. Preprint, available at http://www.math.wustl.edu/ feres/publications.html via link for Les Diablerets minicourse.

[10] Fisher, D., Whyte, K., Continuous quotients for lattice actions on compact spaces, Geom. Dedicata, 87 (2001), no. 1-3, 181-189.

[11] Fisher, D., Zimmer, R. J., Geometric lattice actions, entropy and fundamental groups, Comment. Math. Helv. 77 (2002), no. 2, 326-338..

[12] Gromov, M. Rigid transformation groups. in: Géométrie différentielle, D. Bernard and Y. Choquet-Bruhat (eds.), Travaux en cours 33 (1988) 65-139

[13] Iozzi, A. Invariant geometric structures: a nonlinear extension of the Borel density theorem. Amer. J. Math. 114 (1992), no. 3, 627-648

[14] Katok, A. and Lewis, J. Global rigidity results and new examples of volume-preserving actions. Israel J. Math. 93 (1996) 253-280

[15] Varadajan, V.S. Lie groups, Lie algebras, and their representations, Springer-Verlag, New York, 1984.

[16] Zeghib, A. Sur les groupes de transformations rigides: théorème de l'orbite dense-ouverte. [On rigid transformation groups: open-dense orbit theorem] Rigidité, groupe fondamental et dynamique, 169-188, Panor. Synthèses, 13, Soc. Math. France, Paris, 2002.

[17] Zeghib, A. On Gromov's theory of rigid transformation groups: a dual approach. Ergodic Theory Dynam. Systems 20 (2000), no. 3, 935-946.

[18] R.J. Zimmer, Actions of semisimple groups and discrete subgroups, Proc. Internat. Cong. Math., Berkeley, 1986, 1247-1258.

[19] Zimmer, R. J. Automorphism groups of geometric manifolds. Proc. Symp. Pure Math., v. 54 (1993), pt. 3, 693-710 
E. Jerome Benveniste

Highbridge Capital Inc.

$9 \mathrm{~W} 57$ ST., 27TH FL.

NeW York, NY 10019

jeromeb@hcmny.com

DAVID FISHER

Lehman College - CUNy

Department of Mathematics and Computer Science 250 BedFord Park Boulevard W

BRONX, NY 10468

david.fisher@lehman. cuny.edu

Received October 10, 2002. 
